\title{
Return to Driving After Hip and Knee Arthroscopic Procedures: A systematic review and meta- analysis
}

\author{
Samantha Palma ${ }^{1}$, Purva Patel ${ }^{1}$, Jeya Palan², HG Pandit ${ }^{3}$, BH van Duren ${ }^{3}$ \\ ${ }^{1}$ Indiana University School of Medicine; ${ }^{2}$ Leeds Teaching Hospitals NHS Trust; ${ }^{3}$ University of \\ Leeds, Leeds Institute of Rheumatic and Musculoskeletal Medicine, Chapel Allerton Hospital;
}

Background: Hip and knee arthroscopies are common orthopaedic procedures. As patients are looking to return to their regular schedules and regain their independence post-surgery, physicians often encounter the question of, "when can I drive again?" While safety of the patient is of the utmost importance when making these recommendations, it is equally important to consider the possibility of harm to others and potential legal ramifications. The purpose of this study is to consolidate evidence from available literature and undertake a systematic review and meta-analysis to determine when it is safe for patients to return to driving after hip and knee arthroscopic procedures.

Methods: A systematic review was conducted using PRISMA guidelines. OVID, EMBASE, and COCHRANE databases were searched through June 2020 for articles containing keywords and/or MeSH terms "Hip arthroscopy" and "knee arthroscopy" in conjunction with "total brake response time" or "reaction time" in the context of automobile driving. Title review and full article review were done to assess quality and select relevant articles. Review Manager Version 5.4 was utilized for statistical analysis.

Results: 8 papers were included in the meta-analysis of Brake Reaction Time (BRT). Metaanalysis of all Knee BRTs showed times slower-than or equal-to-baseline BRTs through 5 weeks, with a trend of improving BRT from 6 to 10 weeks (only weeks 8 and 10 were significant $P<$ 0.05). Of all Hip BRTs, week 2 showed slower-than-baseline BRTs, but after week 4 demonstrated a trend toward faster BRTs through week 8 (only week 8 was significant $P<$ $0.05)$.

Conclusion: BRTs met baseline/control values and continued to improve after 6 weeks following knee arthroscopy and after 4 weeks following hip arthroscopy. Based on these results it would be safe to recommend return to driving at 6 weeks after knee arthroscopy and 4 weeks after hip arthroscopic procedures. 